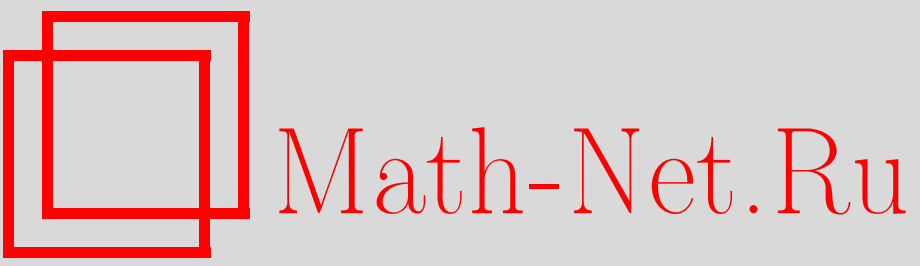

А. В. Арутюнов, О вещественных квадратичных формах, аннулирующих пересечение квадрик, УМH, 2005, том 60, выпуск 1, 161-162

DOI: https://doi.org/10.4213/rm1393

Использование Общероссийского математического портала Math-Net.Ru подразумевает, что вы прочитали и согласны с пользовательским соглашением

http://www.mathnet.ru/rus/agreement

Параметры загрузки:

IP: 3.93 .64 .190

26 апреля 2023 г., 12:44:46 


\title{
О ВЕЩЕСТВЕННЫХ КВАДРАТИЧНЫХ ФОРМАХ, АННУЛИРУЮЩИХ ПЕРЕСЕЧЕНИЕ КВАДРИК
}

\author{
А. В. АРутюнов
}

Пусть даны $k$ квадратных $n \times n$ вещественных симметричных матриц $Q_{i}, i=\overline{1, k}$. Они определяют на $\mathbb{R}^{n}$ квадратичные формы $q_{i}: q_{i}(x)=\left\langle Q_{i} x, x\right\rangle$. Рассмотрим конус

$$
K=\left\{x \in \mathbb{R}^{n}: q_{i}(x) \leqslant 0, i=\overline{1, k_{1}}, q_{i}(x)=0, i=\overline{k_{1}+1, k}\right\}
$$

$\left(n, k\right.$ и $k_{1} \leqslant k$ заданы).

Пусть $Q_{0}$ - заданная вещественная симметричная матрица. Она определяет квадратичную форму $q_{0}(x)=\left\langle Q_{0} x, x\right\rangle$. Будем говорить, что форма $q_{0}$ аннулирует $K$, если $q_{0}(x)=0 \forall x \in K$.

Приведем условия, при которых для любой квадратичной формы $q_{0}$, аннулирующей $K$, выполняется

$$
\exists \lambda \in \Lambda: \quad Q_{0}=\lambda Q, \quad \text { где } \Lambda=\left\{\lambda=\left(\lambda^{1}, \ldots, \lambda^{k}\right) \in \mathbb{R}^{k}: \lambda^{i} \geqslant 0, i=\overline{1, k_{1}}\right\} .
$$

Здесь и ниже $\lambda Q=\sum_{i=1}^{k} \lambda^{i} Q_{i}$.

Введем необходимые обозначения. Для целого неотрицательного $s$ положим $\Lambda_{s}=\{\lambda \in \Lambda$ : ind $\lambda Q \leqslant s\}$. Здесь ind - индекс квадратичной формы (т.е. количество ее отрицательных собственных значений). Через conv обозначим выпуклую оболочку множества и положим $q(x)=$ $\left(q_{1}(x), \ldots, q_{k}(x)\right)$.

ТеОрема. Пусть конус conv $\Lambda_{2(k-1)}$ является острым (т.е. не содержит ненулевых подпространств) и

$$
\exists h \in \mathbb{R}^{n}: \quad\langle q(h), \lambda\rangle<0 \quad \forall \lambda \in \Lambda_{k-1}, \lambda \neq 0 .
$$

Тогда для любой квадратичной формы $q_{0}$, аннулирующей $K$, выполняется (1).

ДокАЗАТЕЛЬСтво. В силу леммы 2 из [1] (см. также [1; с. 23]), (2) и остроты конуса $\operatorname{conv} \Lambda_{2(k-1)}$

$$
\exists \widehat{h} \in \mathbb{R}^{n}:\langle\lambda, q(\widehat{h})\rangle<0 \quad \forall \lambda \in \Lambda_{2(k-1)}, \lambda \neq 0 .
$$

Рассмотрим экстремальную задачу

$$
q_{0}(x) \rightarrow \min , \quad q_{i}(x) \leqslant 0, \quad i=\overline{1, k_{1}}, \quad q_{i}(x)=0, \quad i=\overline{k_{1}+1, k} .
$$

Минимум в ней достигается в анормальной точке $x=0$ (так как по условию $q_{0}(x)=0 \forall x \in K$ ). Применяя к задаче (4) необходимые условия второго порядка (см. [2; $§ 1.4]$, [3], а также [4]), получаем существование таких не равных одновременно нулю $\lambda^{0} \geqslant 0, \lambda \in \Lambda$, что $\operatorname{ind}\left(\lambda^{0} Q_{0}+\lambda Q\right) \leqslant$ $k-1, \lambda^{0} q_{0}(\widehat{h})+\langle\lambda, q(\widehat{h})\rangle \geqslant 0$. Тогда $\lambda^{0}>0$. (Действительно, если $\lambda^{0}=0$, то $\lambda \neq 0$, ind $\lambda Q \leqslant$ $k-1,\langle\lambda, q(\widehat{h})\rangle \geqslant 0$, что противоречит (3).) Поэтому, не теряя общности, можно взять $\lambda^{0}=1$, и, значит,

$$
\operatorname{ind}\left(Q_{0}+\lambda Q\right) \leqslant k-1, \quad q_{0}(\widehat{h})+\langle\lambda, q(\widehat{h})\rangle \geqslant 0 .
$$

Аналогично, заменяя в рассмотренной выше задаче форму $q_{0}$ на форму $\left(-q_{0}\right)$, получаем существование $\bar{\lambda}$ :

$$
\operatorname{ind}\left(-Q_{0}+\bar{\lambda} Q\right) \leqslant k-1, \quad-q_{0}(\widehat{h})+\langle\bar{\lambda}, q(\widehat{h})\rangle \geqslant 0 .
$$

Покажем, что $\bar{\lambda}=-\lambda$. Предположим противное: $\lambda+\bar{\lambda} \neq 0$. Тогда, учитьвая, что индекс суммы квадратичных форм не превосходит суммы их индексов, имеем $(\lambda+\bar{\lambda}) \in \Lambda_{2(k-1)}$,

Работа выполнена при поддержке Российского фонда фундаментальных исследований (грант № 02-01-00334). 
$\langle(\lambda+\bar{\lambda}), q(\widehat{h})\rangle \geqslant 0$. Получили противоречие с (3). Таким образом, $\bar{\lambda}=-\lambda$. Поэтому, положив $\widetilde{Q}_{0}=Q_{0}+\lambda Q$, окончательно имеем:

$$
\text { ind } \widetilde{Q}_{0} \leqslant k-1, \quad \operatorname{ind}\left(-\widetilde{Q}_{0}\right) \leqslant k-1, \quad q_{0}(\widehat{h})+\langle\lambda, q(\widehat{h})\rangle=0 .
$$

Докажем, что $\widetilde{Q}_{0}=0$. Предположим противное. Тогда $\widetilde{q}_{0} \neq 0$, где $\widetilde{q}_{0}(x)=\left\langle\widetilde{Q}_{0} x, x\right\rangle$. Поэтому в силу (3) существует $\widetilde{h}$, достаточно близкое к $\widehat{h}$, для которого

$$
\langle\mu, q(\widetilde{h})\rangle<0 \quad \forall \mu \in \Lambda_{2(k-1)}, \quad \widetilde{q}_{0}(\widetilde{h}) \neq 0 .
$$

Проводя рассуждения, аналогичные приведенным выше, но уже не для $\widehat{h}$, а для $\widetilde{h}$, получаем, что существует $\widetilde{\lambda}$ такое, что

$$
\operatorname{ind}\left(\widetilde{Q}_{0}+\widetilde{\lambda} Q\right) \leqslant k-1, \quad \operatorname{ind}\left(-\left(\widetilde{Q}_{0}+\widetilde{\lambda} Q\right)\right) \leqslant k-1, \quad\left\langle\left(\widetilde{Q}_{0}+\widetilde{\lambda} Q\right) \widetilde{h}, \widetilde{h}\right\rangle=0 .
$$

Из первой формулы в (5) и второй формулы в (7) имеем

$$
2(k-1) \geqslant \operatorname{ind}\left(\widetilde{Q}_{0}-\left(\widetilde{Q}_{0}+\widetilde{\lambda} Q\right)\right)=\operatorname{ind}(-\widetilde{\lambda} Q) \Longrightarrow \operatorname{ind}(-\widetilde{\lambda} Q) \leqslant 2(k-1) .
$$

Аналогично из второй формулы в (5) и первой формулы в (7) получаем ind $\widetilde{\lambda} Q \leqslant 2(k-1)$. Таким образом, $\tilde{\lambda} \in \Lambda_{2(k-1)},-\widetilde{\lambda} \in \Lambda_{2(k-1)} \Longrightarrow \widetilde{\lambda}=0$, так как по условию конус conv $\Lambda_{2(k-1)}$ острьй. Но из равенства в $(7)$ в силу $\widetilde{\lambda}=0$ имеем $\widetilde{q}_{0}(\widetilde{h})=0$, что противоречит $(6)$. Полученное противоречие доказывает, что $\widetilde{Q}_{0}=0 \Longrightarrow Q_{0}+\lambda Q=0$. Теорема доказана.

Отметим, что если $k_{1}=k$, то $\Lambda$ - это положительный ортант и поэтому конусы $\Lambda$, а значит, и conv $\Lambda_{2(k-1)}$, являются острыми. Если $n \gg k$, то для квадратичных отображений $Q$ общего положения конус conv $\Lambda_{2(k-1)}$ является острым [5].

\section{СПИСОК ЛИТЕРАТУРЫ}

[1] А. В. Арутюнов // Матем. сб. 2000. Т. 191. № 1. С. 3-26. [2] А. В. Арутюнов. Условия экстремума. Анормальные и вырожденные задачи. М.: Факториал, 1997. [3] А. В. Арутюнов, В. Ячимович // Вестник Моск. ун-та. Сер. 15, выгисл. матем. и кибернет. 1999. № 4. С. 34-40. [4] А. С. Матвеев. Критерии выпуклости образов квадратичных отображений в теории оптимального управления системами, описываемыми дифференциальными уравнениями // Автореферат дисс. ... докт. физ.-матем. наук. С.-Петербург: СПбГУ, 1998. [5] А. А. Аграчев // Итоги науки и техники. Сер. Алгебра. Геометрия. Топология. Т. 26. М.: ВИНИТИ, 1988. C. $85-124$. 